\title{
Frequency Analysis of Grid Connected EVS by using Artificial Neural Network (ANN)
}

\author{
Ranjith Kumar Reddy Banda ${ }^{1}$, Karunakumar Davala ${ }^{1}$, Dola Gobinda Padan $^{1}$, Anil Kumar Rajagiri ${ }^{1}$
}

${ }^{1}$ EEE Department, GRIET, Hyderabad

\begin{abstract}
The vehicle-to-grid (V2G) model is able to provide the power-systems that have been built to incorporate the hybrid electric vehicle model on a wide scale with distributed reserve. The authors suggested an amended V2G control model that would concurrently manage different renewable power sources, vehicle idle time and electricity generation on a vehicle consumer day basis. In respect of the desired status of battery and the detected plug-on terminal, vehicle-to-grid power is tested. This article presents an intelligent decision- making system based on an artificial neural network (ANN) that uses data logged by the M2MAMI for the planning and management of electricity charge. The ANN has been trained with household energy usage and EV energy requires the data and convention to determine when to charge the vehicle (G2V) or to discharge it (V2G). Charge Terminology, Electric Cars, Energy storage, Neural Network. Charge Scheduling. In this paper, MATLAB/Simulink implements the proposed control block. Different virtual images evaluate the performance of the control structure, interface, communications, device efficiency and time responses.
\end{abstract}

\section{Introduction}

Cars offered a comfortable lifestyle to society. Personal cars are preferred for freedom, protection and comfort over other modes, whereas travelling along greater distances is suitable for rail, aviation, etc. Without these carriages, animals would have drawn humans. Inventions, such as mechanical vehicles, balloon vehicles, steam-powered vehicles, push bikes, flights, helicoppers, aircraft etc. have reached up to one billion since 1350, in earliest known railway record. While these transport modes have generated many businesses, the environmentalimpacts they have had are devastating. And before they start moving, they use a lot of resources. The consumptive fuel and waste of the greenhouse, as well as other gases causing pollution such as carbon monoxide, nitrogen oxides and etc., are the main risks as the oxides of nitrogen and the hydrocarbons are reacting together trendy the eventof sunlight leading to the rise in the ground-level ozone. The transport sector primarily includes domestic combustion vehicles with an energy efficiency of up to $20 \%$.

The other big effect of transportation is the mixture of petroleum in much of the world, such as environmental concern for fuel consumption, high petroleum prices and the potential for peak petroleum. In the mid-19th century, one solution was found to the

\footnotetext{
*Ranjith Kumar Reddy Banda: bandaranjithreddy93@gmail.com
}

threat posed by automobiles. This led to the demand for production of the cleaner alternative fuel or unconventional vehicle power systems. Although internal combustion engines remained the predominant motor vehicle power system for the next 100 years, electricity was used in different types of trains and smaller vehicles. Due to technological advances the electric vehicles (EVs) began to emerge as the burden of renewable energy was to change. When people came up with the concept, demand grew and engineers started sharing technical information to make electricity conversions[2-3]. Different kinds of EVs classified as their energy sources by the degree of electricity have appeared in industry. The major classes include: Electric Vehicle Batteries (BEV), Electric Hybrid Vehicles (HEVs) (PHEVs). Petrol and electricity are powered by HEVs. Regenerative braking produces the electric power to be used.This is a mechanism that helps to slow the vehicle down by the electric motor. It uses the energy transferred by the brakes to heat. You begin using the electric engine, followed by the gasoline engine as the cargo or the speed increases. The two engines are regulated by an internal computer that ensures the best driving performance. PHEVs are the same as HEVs, only distinction lying in another charge mode, namely, relation to the external power supply. The petrol engine expands the range of the car and when it gets low the battery also recharges. BEVs are completely EVs; i.e. they are only powered by electricity and have no fuel, oil only powered by electricity and have no fuel, oil or exhaust tube. They load either through 
the regenerative braking or through an external electric charge outlet. EVs also make it possible for delivery services to receive demand dispatch advantageously by [4-9].

The demand for electricity is enormous if a company has more EVs on the road than motor vehicles. Various renewable energy farms can be used, excluding the diesel generator, for the production of electricity, such as Photovoltaic (PV), wind farming, hydraulic farms, etc. Besides, an idea on the use of power in an idle vehiclecontributed to the creation of a technology to consume and generate smarter electricity. Micro- networks are clusters and their power loads from local generation sources. An idea was a "smart grid" that introduced an intelligent electricity micro grid network that would track, diagnose and re- equalize itself to increase performance. This aims to prevent or mitigate the situation of blackouts. The previously proposed grid system operated on transfers of power from the generator into the car, while this idea of intelligent grids differs because it enables 2-way communication and power transmission. This function makes additional controls, grid functions and efficiency [1011]. They are designed to accommodate all generation and storage sources of electricity which reducesenergy use and costs, delivers high-quality power and the reliability, enables controls, self-healing and provides power and programming participation for end-users. Here, summarised idea is grid power storage and transferred back to the vehicle when required, and when the vehicle is idle it is returned to the grid. In addition to savings for energy bills, this reduces unwanted demand curve peaks occurring at a certain time of the day, leading to more favourable restructuring [12-13].

The authors have introduced an ANN network in [14] to forecast the use of hourally-built buildings and the production of solar electricity in order to establish an optimum charging schedule. However, they did not clearly mention when V2G / G2V operations were needed for the EV. In [15] the authors trained an ANN using power transformers, current and charging status of the EV (SoC) and number of vehicles are predicted that after an EV interaction the current and the voltage will be changed. They did not inquire when the vehicle had to be loaded or unloaded. We take a step forward here and decide whether an EV can load, unload or stay idle with a trained ANN.

\section{EV Configuration}

This model illustrates the network that regulates the microgrid frequency for 24-hour events[14]. A model displays a vehicle network. The proposed micro grid consists of four main components.

i) the main source of power generation diesel, ii) the wind farm combined with the photovoltaic farm, iii) V2G system, bidirectional block and household load iv) to satisfy overhead energy source requirements.

This micro grid supports thousands of households during the windy day in the summer. A model of a V2G consists of 100 electric cars, which provide a ratio of 1:10 to cars and households. A diesel generator compensates for the disparity between renewable and used energy and the V2G. First is an energy-proportionate photovoltaic farm, with an energygenerated proportion of three factors for a micro-survey of a couple of renewable sources: the location of a source of energy, panel efficiency and irradiation detail. When the wind reaches the nominal value, a power station produces nominal power. If wind speed is above the highest permissible value and a wind comes down to its nominal value, the wind farm cuts the grid.

Vehicle-to-Grid (V2G) device mathematicalmodelling: Equation can be used for the electricity used by electric vehicles:

$P_{E V}(\mathrm{t})=P_{\text {max }}\left\{1-e^{-\left(\alpha, t / t_{\max }\right.}\right\}+P_{E V_{o}}$

The demand of power is:

$P_{\text {dem }}(\mathrm{t})=\mathrm{n} . P_{\max }-P_{\max } \sum_{i=1}^{n} e^{-\left(\propto_{i} n_{r} t_{c i} / t_{\max }\right)}$

The dissipated power is evaluated as:

$P_{\text {export }}(\mathrm{t})=\sum_{i=1}^{n} P_{E V_{o i}}\left(1-e^{n_{i} \beta_{i} t_{s i} / t_{\text {max }}}\right)$

Therefore, the net power is:

$P_{n e t}(\mathrm{t})=\sum_{i=1}^{n} P_{E V_{o i}}\left(1-e^{n_{i} \beta_{i} t_{s i} / t_{\max }}\right)-P_{\max }\left(n_{d}-\right.$

$\sum_{i=1}^{n} e^{-\left(\propto_{i} n_{r} t_{c i} / t_{\max }\right)}$

The V2G has two functions: to control the battery charge and to use the power to control the grid if there is a regular case.

Five entirely different user profiles are implemented in this block:

\#1: people who come to work in the car loading facility

\#2: people who deal with the installation but who load their cars for a long journey Profile:

\#3: people going to work for a Profile of their cars without any facilities

\#4: people staying at home profiles during the day

\#5: people to work on a shift at night

The load includes residential loads on the micronet. It follows a specific power level consumer profile. It takes 24 hours for a simulation to take place. The strength of light follows a normal distribution and during mid-day it can be observed. The wind variesconsiderably all day and has many peaks and lower peaks. The residential load is more like normal consumption in a city following a traditional pattern. Consumption is low all day long and will rise until the peak in the night and decrease slowly in the afternoon.

The main events influencing the frequency of thegrid were:

1. High noon partial shading eliminates for a specific time one renewable (alternative) energy source.

2. Wind power supply to be cut off at 22 hours as the maximum allowable mark reaches the wind levels. The MATLAB V2G model is shown in Fig.1. 


\section{Methodology}

This paper refers to several changes in a Simulink model. a model has been extensively examined and a following control structures have undergone changes: Load: a residential load power factor used in an original model was 0.15 ; it was updated and raised to 0.95 resulting in higher energy efficiency and lower losses. V2G: All car profiles have been changed to progressively change a charge status (SOC). a SOCs dropped to the rate of 0.1 units per hour during flight, i.e. plug-state 0 . a SOCs remained unaltered if a plug condition was 1 and a settled SOCs increased and reached the limit of 0,95 and a grid was charged when an energy farm was over consumption or sudden closure. a SOC calculation control block was changed, which corresponds to a decrease of a SOC of HEV used ina plug state, and has the SOC variable during a period of a plug state of a specific HEV. a traditional and modified control strategy is represented in Fig.2 and 3 .

\section{Artificial Neural Network}

Artificial neurons consist of inputs (similar to a dendrites), which are multiplied by weights (strength of a signals concerned) (activation function). a major challenge for an ANN to achieve a desired result for such inputs, however, is to change weights of an artificial neurons. Some algorithms are used to modify ANN weights in order to achieve a desired performance. Learning or training is a method of changing a weights of ANN. Since learning requires several thousand weights change, learning is the technique of optimisation to minimise an overall error function[16]. the low pass filter for the weighted sum of a neuron inputs is an active/transfer function to generate a required output within the limited range[18] This function operates as the small pass filter. Figure 1 displays different network training activation functions.

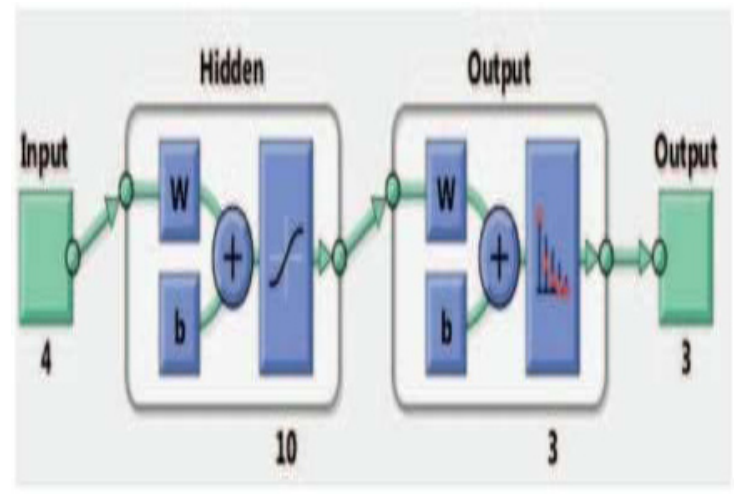

Fig.1. Neural Network for Training and Data Validating for this Research.
We used the supervised feed-forward neural network in this research. a supervision is because a trained network will generate a desired outputs as the response to sample inputs and enable us to see how an actual outputs correspond closely with those desired and makes it especially suitable for dynamic system modelling and control, classification of noisy data and future events prediction. a term feed-forward on another hand means that information propagates between an inputs and outputs without any feedback process, and that between an input and output layers may be "multistage," that of one or more hidden layers. We use a back propagation algorithm of a scaled conjugated gradient (SCG) to train a network.

We used the 2-layer feed-forward network, consisting of hidden-layer sigmoid neurons, and output-layer soft-max [19] neurons capable of classifying vector, particularly when V2G or G2V is needed. We used real data covering two days of electricity use, travel time, charging status (SoC) in network preparation. an ANN performance indicates a recommended operating mode of an $\mathrm{EV}$, i.e. V2G, G2V or idle as the time feature.

smart metre and from 3 to 288 . These samples were divided into $70 \%$ for preparation, $15 \%$ for validation and the remaining ones for research.

\section{Results}

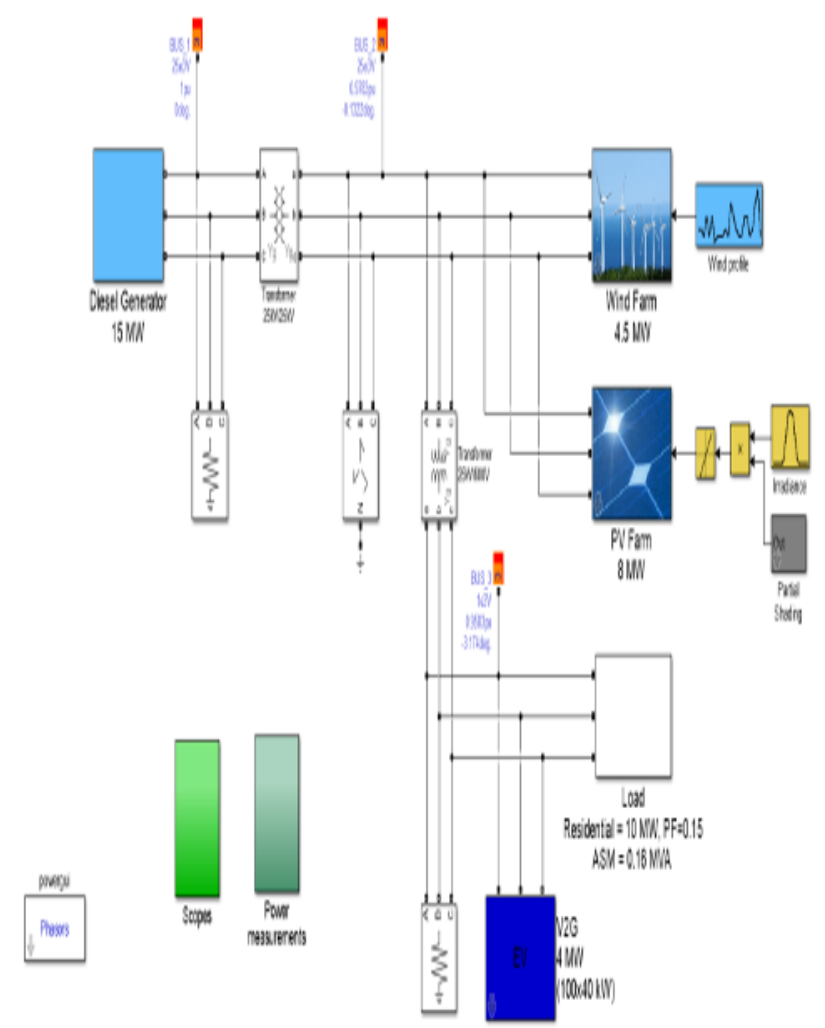

Fig.2. Simulation diagram of V2 


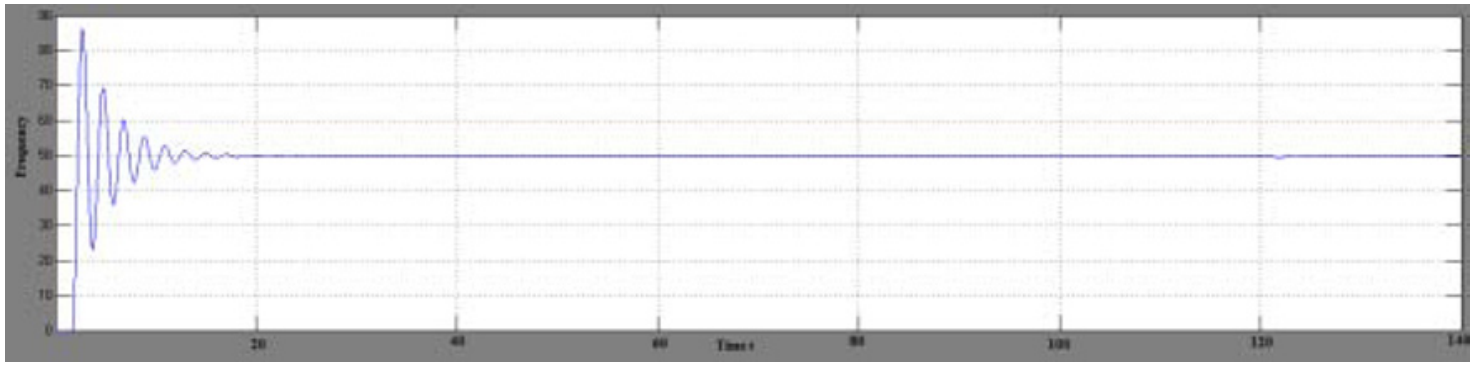

Fig.3. Frequency deviation with PI controller

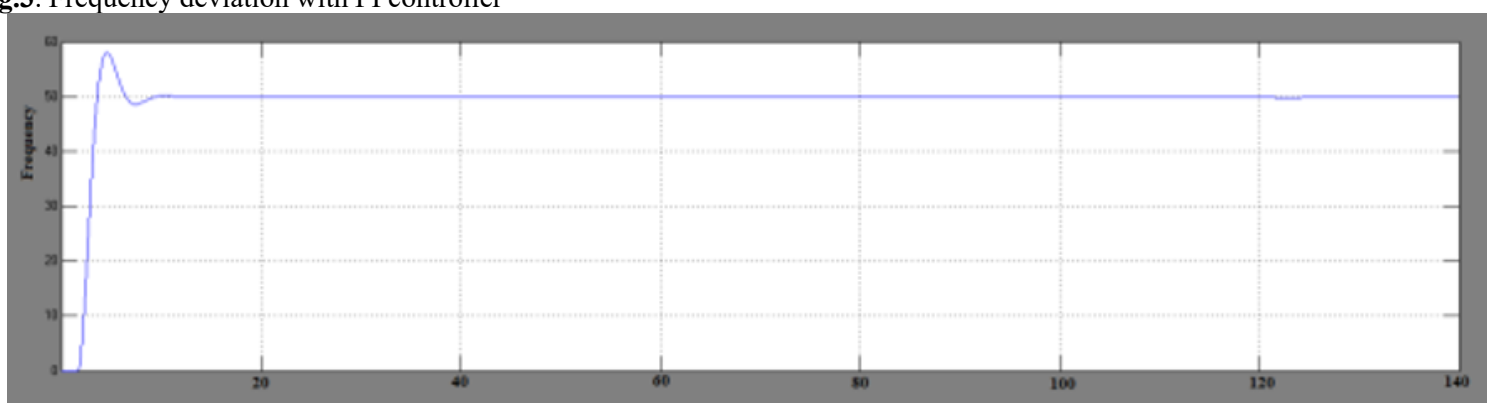

Fig.4. Frequency deviation with ANN controller

\section{Conclusion}

A V2G model is built with different energy renewables, vehicle operating times and electricity generation. By incorporating time delays and thus incremental improvements, vehicle-to-grid transitions were made possible. The activity of the model was completed with the addition of functions corresponding to the plug status 0 ,including tagging of a profile as the "control mode" or "charge mode". It took more than initial time to charge. These changes also increased the reliability of the power supply. The other scopes include changes to the PI controller, which includes intelligence to better analyse the V2G model in realtime. The ANN is to be included in the scheduling decision by two areas of further work which is being investigated and repetitive ANNs to incorporate those predictive capacities in the decisionmaking schedule.

\section{References}

1. Chen Jie1, Zhang Yueyu, SU Wencong,China Comm,( 2015).

2. A. Brooks, E. Lu, D. Reicher, C. Spirakis, B.Weihl IEEE Pow. Engy. Mag., vol. 8, pp..20-29, (2010)

3. D.S. Callaway, I.A. Hiskens, Proc. IEEE, vol. 99 , pp. 184-199, (2011).

4. J.A.P. Lopes, F.J. Soares, P.M.R. Almeida, in Proc. IEEE, vol. 99, iss.1, 2011, pp.168-183,(2011).

5. M.Esmaili, M.Rajabi,IET Gen.Trans..Distr. pp 583-590,(2014)
6.K. Clement-Nyns, E. Haesen, J. Driesen, IEEETrans.Pow. Sys.25, pp.371-380(2010).

7.E. Sortomme, M.M. Hindi, S.J. MacPherson, S. Venkata, , IEEE Trans. Smart Grid, vol. 2, pp. 198-205, (2011).

8. S.Deilami, A.S. Masoum, P.S. Moses, M.A. Masoum, IEEE Trans. Smart Grid, vol. 2, pp.456-467, (2011).

9. D. Oliveira, A.Z. de Souza, L. Delboni, Elec. Pow. Sys. Res., 98, pp.77-85, (2013).

10. Rahimi F, Ipakchi.A,IEEE Trans Smart Grid, 1, pp. 8288,(2010).

11. Palensky P, Dietrich.D.IEEE Trans Ind., 7 pp. 381-388, (2011).

12. Incorporing demand side flexibility, in particular demand response, in electricity markets" EU Commission Staff Working Document, 2013.

13. Logenthiran T, Srinivasan D, Shun TZ, IEEE Trans. Smart Grid, 3, pp. 1244-52, (2012). 